\title{
Pairing correlations and symmetries in odd-A nuclei.
}

\author{
J. Luis Egido ${ }^{1, *}$ and Marta Borrajo ${ }^{1}$ \\ ${ }^{1}$ Departamento de Física Teórica, Universidad Autónoma de Madrid, E-28049 Madrid, Spain
}

\begin{abstract}
The pairing correlations in odd-A nuclei are analyzed in the mean field approximation and beyond. In particular the role of symmetry conservation is investigated. We find that particle number projection after the variation (PN-PAV) has little effect on the pairing correlations specially in the weak pairing regime. This is in contrast to the variation after particle number projection (PN-VAP) approach where a strong effect is found. The situation is specially critical in odd nuclei because the pairing correlations vanish due to the blocking effect and the Hartree-Fock-Bogoliubov wave function collapses to the Hartree-Fock one. The PN-VAP, however, handles perfectly the exact blocking providing highly correlated wave functions. The role of the angular momentum projection is studied only in the PAV approach. We find small changes of the pairing correlation, at least at small angular momentum. In the calculations we use the Gogny interaction well suited to this kind of studies.
\end{abstract}

\section{Introduction}

Pairing correlations play a predominant role in the description of nuclear phenomena [1]. Basic approaches like the mean field (MF) only provide a realistic description when the superfluidity is incorporated into the theory. The success of the BCS approach and the Hartree-FockBogoliubov (HFB) theory are good examples of it. The consideration of pairing correlations provides a qualitative difference in the description of basic properties like oddeven mass differences, spectra of even-even and odd-even A nuclei and dynamical masses -e.g. moments of inertiato mention a few. The great breakthrough of the BCS was to consider the symmetry breaking mechanism to include particle-particle correlations in a product (of quasiparticles) wave function. Another example of the fruitful idea is the angular momentum. In this case the breaking of the rotational symmetry allows to describe deformed nuclei by simple product wave functions.

BCS and HFB theories, however, have some drawbacks like the pairing collapse in the weak pairing regime and the non-conservation of the particle number (PN) and angular momentum (AM) symmetries. To overcome these problems and at the same time to benefit from the simplicity of product wave functions the symmetry conserving mean field (SCMF) approach has been developed [2-4]. In this theory use is made of the projectors technique to obtain eigenstates of the particle number operator and the angular momentum from symmetry breaking HFB wave functions [5]. At this point it is important to recall the two ways to recover a symmetry. They differ in the way the matrices $(U, V)$ of the Bogoliubov transformation, see Eq. (1) below, are determined. The simplest way is the so-called projection after variation (PAV). In this approach the matrices $(U, V)$ are determined by minimizing the HFB

\footnotetext{
*e-mail: j.luis.egido@uam.es
}

energy and only afterwards the HFB wave function is projected to calculate the different observables. In the more demanding variation after projection (VAP), $(U, V)$ are determined by minimizing the projected energy. Obviously only the latter one provides a selfconsistent determination of $(U, V)$ compatible with the symmetries of the system. An inherent failure of the PAV approach is that the pairing collapse in the weak pairing cases cannot be avoided.

The SCMF wave funtions are the building blocks of the modern beyond mean field (BMF) approaches recently developed with the Skyrme, Gogny and relativistic effective interactions [6-9]. In these theories a basis of SCMF wave funtions is provided by the generator coordinate method (GCM) using as coordinates the most relevant nuclear degrees of freedom [10-15]. The Hamiltonian eigenstates are developed in this basis and the mixing coefficients determined by the variational principle. These developments are called symmetry-conserving configuration mixing (SCCM) approaches and have been applied mostly to even-even nuclei. Attempts to extend these approaches to odd-even and odd-odd nuclei are underway [16-18]. This task is not easy because odd nuclei are far more complicated to deal with. Even at the mean-field level like in the HFB theories, odd nuclei are numerically cumbersome and to calculate ground states one must try several spins, parity, etc. Furthermore, the blocked structure of the wave function entails the breaking of the timereversal symmetry and triaxial calculations must be performed. An important problem not to be overlooked in SCCM calculations is the fact that the blocked structure of the wave function favors the quenching of the pairing correlations. That means, if in some basis states of the GCM linear combination the pairing collapses, the dynamical correlations introduced by the diagonalization of the associated Griffins-Hill-Wheeler equation will be wrong. To investigate this fact in this paper we study the even-odd 
nucleus ${ }^{27} \mathrm{Mg}$ in the SCMF approach using as generator coordinates the quadrupole deformation parameters $(\beta, \gamma)$ in the PAV and VAP approaches. In the calculations we use the Gogny interaction $[19,20]$, which is well suited to deal with the pairing correlations.

\section{Theory}

Our starting approach is the HFB theory [5]. As a meanfield approximation the HFB wave function $|\phi\rangle$ is a product of quasi-particles $\alpha_{\rho}$ defined by the general Bogoliubov transformation

$$
\alpha_{\rho}^{\dagger}=\sum_{\mu} U_{\mu \rho} c_{\mu}^{\dagger}+V_{\mu \rho} c_{\mu},
$$

where $c_{\mu}^{\dagger}, c_{\mu}$ are the particle-creation and -annihilation operators in the reference basis, in our case the Harmonic Oscillator one. $U$ and $V$ are the Bogoliubov matrices to be determined by the Ritz variational principle. In our approach we have imposed the simplex, $\Pi_{1}=\hat{P} e^{-i \pi J_{x}}$, which allows to characterize the blocking structure of odd and even nuclei $[2,21]$. The index $\mu$ runs over the states $\{1,2, \ldots, M, \overline{1}, \overline{2}, \ldots, \bar{M}\}$. The states $\alpha_{m}^{\dagger}$ have simplex $+i$ and $\alpha_{\bar{m}}^{\dagger}$ have $-i$.

The wave function of the ground state of an even-even nucleus is given by ${ }^{1}$

$$
|\phi\rangle=\prod_{\mu=1}^{2 M} \alpha_{\mu}|-\rangle=\prod_{\mu=1}^{M} \alpha_{m} \alpha_{\bar{m}}|-\rangle,
$$

with $|-\rangle$ the particle vacuum. The quasiparticle vacuum $|\phi\rangle$ is defined by

$$
\alpha_{\mu}|\phi\rangle=0, \quad \mu=1, \ldots, 2 M,
$$

and has simplex +1 . The quasiparticle excitations

$$
|\tilde{\phi}\rangle=\alpha_{\rho_{1}}^{\dagger}|\phi\rangle
$$

correspond to odd-even nuclei. They can be written as vacuum to the quasiparticle operators $\tilde{\alpha}_{\rho}$,

$$
\tilde{\alpha}_{\rho}|\tilde{\phi}\rangle=0, \quad \rho=1, \ldots, 2 M .
$$

The $2 M$ operators $\left\{\tilde{\alpha}_{\rho}^{\dagger}\right\}$ are obtained from the set $\left\{\alpha_{\mu}^{\dagger}\right\}$ by replacing the creation operator $\alpha_{\rho_{1}}^{\dagger}$ by the annihilation operator $\alpha_{\rho_{1}}$, the other $2 M-1$ operators remain unchanged. In this work we are interested in the nucleus ${ }^{27} \mathrm{Mg}$. We therefore consider wave functions of the form

$$
\left|\tilde{\phi}^{\pi}\right\rangle=\alpha_{\rho_{1}}^{\dagger} \prod_{\mu=1}^{2 M} \alpha_{\mu}|-\rangle
$$

with $\rho_{1}$ a neutron state. Notice that in the running product of Eq. (6), orbitals with the same parity are occupied pairwise, therefore the parity, $\pi$, and the simplex of the state $\left|\tilde{\phi}^{\pi}\right\rangle$ are those of the blocked level $\alpha_{\rho_{1}}^{\dagger}$. One can, of course,

\footnotetext{
${ }^{1}$ The quasiparticle operators that annihilate trivially the particle vacuum are to be omitted from the product.
}

block a state with positive or negative simplex, but since we do not break time reversal explicitly both possibilities are degenerated.

Though the state $|\tilde{\phi}\rangle$ has the right blocking structure, since the Bogoliubov transformation mixes creator and annihilator operators and states with different angular momenta, $|\tilde{\phi}\rangle$ is neither an eigenstate of the particle number nor of the angular momentum operators. As with eveneven nuclei, to recover the symmetries one has to project to the right quantum numbers, see [5]. In the spirit of the SCMF approach, our ansatz is

$$
\left|\Psi_{M, \sigma}^{N, I, \pi}\right\rangle=\sum_{K} g_{K \sigma}^{I} P^{N} P_{M K}^{I}\left|\tilde{\phi}^{\pi}\right\rangle,
$$

the parameters $g_{K \sigma}^{I}$ and the matrices $\{\tilde{U}, \tilde{V}\}$ of the Bogoliubov transformation are determined by the variational principle

$$
\delta E^{N, I, \pi}=\delta \frac{\left\langle\Psi_{M}^{N, I, \pi}\left|\hat{H} \hat{P}^{N} P_{M}^{I}\right| \Psi_{M}^{N, I, \pi}\right\rangle}{\left\langle\Psi_{M}^{N, I, \pi}\left|\hat{P}^{N} P_{M}^{I}\right| \Psi_{M}^{N, I, \pi}\right\rangle}=0 .
$$

This way of solving the SCMF approach is called variation after projection of the particle number and angular momentum, PNAM-VAP. Unfortunately this equation is too complicated to be solved with effective interactions, as in our case, and for large configuration spaces. There are several ways to approximate the exact solution of Eq. (8). The simplest way would be to minimize the HFB energy, i.e., determine $(\tilde{U}, \tilde{V})$ and then perform the projections, i.e. the so-called projection-after-variation (PAV). This method does not provide a good approximation. A much better approach is the following: From eveneven nuclei one knows that PN-VAP (that means, solving Eq. (8) projecting only on the particle number) is feasible while AM-VAP is very CPU-time consuming. The approach of solving the PN-VAP variational equation to find the self-consistent minimum and afterwards to perform an AM-PAV is not very good because the AMP is not able to exploit any degree of freedom of the HFB transformation and self-consistency with respect to the AMP is not guaranteed. An intermediate way is to perform an approximate AM-VAP approach by solving the variational PNVAP equation for a large set of relevant wave functions as to cover the sensitive degrees of freedom. Afterwards an AM-PAV to this set of wave functions will determine the absolute minimum among these states for different angular momenta. We choose the deformation parameters $(\beta, \gamma)$ as the additional degrees of freedom. Notice that this method guarantees, at least, AM-VAP self-consistency with respect to these relevant quantities. Therefore, in order to obtain a grid of wave functions we minimize the PN-VAP constrained energy

$$
E^{\prime}\left[\tilde{\phi}^{\pi}\right]=\frac{\left\langle\tilde{\phi}^{\pi}\left|\hat{H} \hat{P}^{N}\right| \tilde{\phi}^{\pi}\right\rangle}{\left\langle\tilde{\phi}^{\pi}\left|\hat{P}^{N}\right| \tilde{\phi}^{\pi}\right\rangle}-\left\langle\tilde{\phi}^{\pi}\left|\lambda_{q_{0}} \hat{Q}_{20}+\lambda_{q_{2}} \hat{Q}_{22}\right| \tilde{\phi}^{\pi}\right\rangle,
$$

with the Lagrange multiplier $\lambda_{q_{0}}$ and $\lambda_{q_{2}}$ being determined by the constraints

$$
\left\langle\tilde{\phi}^{\pi}\left|\hat{Q}_{20}\right| \tilde{\phi}^{\pi}\right\rangle=q_{0}, \quad\left\langle\tilde{\phi}^{\pi}\left|\hat{Q}_{22}\right| \tilde{\phi}^{\pi}\right\rangle=q_{2} .
$$


The relation between $(\beta, \gamma)$ and $\left(q_{0}, q_{2}\right)$ is given by $\beta=$ $\sqrt{20 \pi\left(q_{0}^{2}+2 q_{2}^{2}\right)} / 3 r_{0}^{2} A^{5 / 3}, \gamma=\arctan \left(\sqrt{2} q_{2} / q_{0}\right)$ with $r_{0}=$ $1.2 \mathrm{fm}$ and $A$ the mass number. The minimization of Eqs. (9-10) is performed with the conjugated-gradient method [22].

The next step is the simultaneous particle-number and angular-momentum projection (PNAMP) of each state $\left|\tilde{\phi}^{\pi}(\beta, \gamma)\right\rangle$ that conforms the $(\beta, \gamma)$ grid,

$$
\begin{aligned}
\left|\Psi_{M, \sigma}^{N, I, \pi}(\beta, \gamma)\right\rangle & =\sum_{K} g_{K \sigma}^{I} P^{N} P_{M K}^{I}\left|\tilde{\phi}^{\pi}(\beta, \gamma)\right\rangle \\
& =\sum_{K} g_{K \sigma}^{I}|I M K, \pi, N,(\beta, \gamma)\rangle,
\end{aligned}
$$

where the coefficients $g_{K \sigma}^{I}$ are variational parameters. They are determined by the energy minimization which provides a reduced Hill-Wheeler-Griffin [23] equation

$$
\sum_{K^{\prime}}\left(\mathcal{H}_{K, K^{\prime}}^{N, I, \pi}-E_{\sigma}^{N, I, \pi} \mathcal{N}_{K, K^{\prime}}^{N, I, \pi}\right) g_{K^{\prime} \sigma}^{I}=0
$$

where $\mathcal{H}_{K K^{\prime}}^{N, I, \pi}$ and $\mathcal{N}_{K, K^{\prime}}^{N, I, \pi}$ are the Hamiltonian and norm overlaps defined by

$$
\begin{aligned}
\mathcal{H}_{K, K^{\prime}}^{N, I, \pi} & =\left\langle I M K, \pi, N,(\beta, \gamma)|H| I M K^{\prime}, \pi, N,(\beta,(\gamma)\}\right) \\
\mathcal{N}_{K, K^{\prime}}^{N, I, \pi} & \left.=\langle I M K, \pi, N,(\beta, \gamma)| I M K^{\prime}, \pi, N,(\beta, \gamma) \chi 14\right)
\end{aligned}
$$

The presence of the norm matrix in Eq. (12) is due to the non-orthogonality of the states $|I M K, \pi, N,(\beta, \gamma)\rangle$. Eq. (12) is solved by standard techniques [5]. Notice that at each $(\beta, \gamma)$ point one can have several eigenvalues $E_{\sigma}^{N, I, \pi}$ labeled by $\sigma$, the lowest solution corresponds to $\sigma=0$.

The solution of Eqs. (12) in the $(\beta, \gamma)$ grid for different angular momenta and parity provides $E_{\sigma}^{N, I, \pi}(\beta, \gamma)$ as a function of $(\beta, \gamma), I, \pi$ and $\sigma$. This energy can be written as

$$
E_{\sigma}^{N, I, \pi}(\beta, \gamma)=\frac{\left\langle\Psi_{M, \sigma}^{N, I, \pi}(\beta, \gamma)|H| \Psi_{M, \sigma}^{N, I, \pi}(\beta, \gamma)\right\rangle}{\left\langle\Psi_{M, \sigma}^{N, I, \pi}(\beta, \gamma) \mid \Psi_{M, \sigma}^{N, I, \pi}(\beta, \gamma)\right\rangle},
$$

which obviously represents the potential energy surface (PES) of the projected energy in the $(\beta, \gamma)$ plane for the given quantum numbers. This projected PES differs from the usual mean field PES and is angular momentum $(I)$, parity $(\pi)$ and state $(\sigma)$ dependent. The minimum value of $E_{\sigma}^{N, I, \pi}(\beta, \gamma)$ in the PES provides the energy and the deformation parameters $\left(\beta_{\mathrm{min}}, \gamma_{\mathrm{min}}\right)$ of the state characterized by the quantum numbers $(I, \pi, \sigma)$ in this approximation. Its wave function is given by $\left|\Psi_{M, \sigma}^{N, I, \pi}\left(\beta_{\min }, \gamma_{\min }\right)\right\rangle$. Notice that by changing the values of $I$ and $\pi$ we can calculate the spectrum built by the lowest states of a given spin and parity.

In the precedent approach to generate the states $\left|\tilde{\phi}^{\pi}(\beta, \gamma)\right\rangle$ we have use the PNVAP approach solving Eq. (9). In this case the pairing degree of freedom is treated fully self-consistently with the conservation of the particle number. This approach is rather time consuming and simpler approximations are often used in the literature to produce the states $\left|\tilde{\phi}^{\pi}(\beta, \gamma)\right\rangle$. The most popular is the constrained HFB approximation in which the HFB energy, given by an expression similar to Eq. (9) but without the projector $\hat{P}^{N}$, is minimized. Two additional constraints on the number of protons and neutrons have to be added to keep the right number of particles at least on the average. We will call this approach the HFB approach. Since the use of the HFB method only affects the wave functions $\left|\tilde{\phi}^{\pi}(\beta, \gamma)\right\rangle$ the rest of the procedure, i.e, the solution of Eqs. 12, is the same in the PNVAP and in the HFB approach. As mentioned in the Introduction is this paper we want to elucidate how good the HFB approach is as compared to the PNVAP in the SCMF and in SCCM calculations.

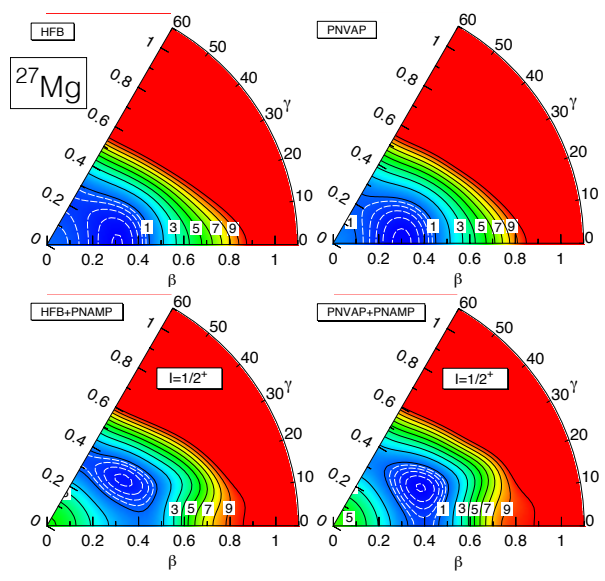

Figure 1. Potential energy surfaces in the $(\beta, \gamma)$ plane for ${ }^{27} \mathrm{Mg}$ in several approaches.

\section{Results}

In the calculations the intrinsic many body wave functions $|\tilde{\phi}(\beta, \gamma)\rangle$ are expanded in a Cartesian harmonic oscillator basis and the number of spherical shells included in this basis is $N_{\text {shells }}=6$ with an oscillator length of $b=1.01 A^{1 / 6}$. The $(\beta, \gamma)$ grid of equilateral triangles contains 116 points. The angular momentum projection has been done with the set of integration points in the Euler angles $\left(N_{\alpha}=N_{\beta}=N_{\gamma}=32\right)$ in the intervals $\alpha \in[0,2 \pi], \beta \in[0, \pi], \gamma \in[0,2 \pi]$. The number of points to perform the integral of the particle-number projection is 11. In the calculations we use the Gogny interaction [19] with the D1S parameterization [20]. We consider all exchange terms of the interaction, the Coulomb force and the two-body correction of the kinetic energy to avoid problems with the PNP [24]. Concerning the density dependence of the force we adopt the projected density prescription for the PNP and the mixed one for the AMP, for further details see for example Refs. [8, 11, 25].

As an example we present in Fig. 1 contour plots of the potential energy surfaces for the nucleus ${ }^{27} \mathrm{Mg}$ calculated in several approaches. In the PN-VAP approach be means of Eq. (9) and in the PNVAP+PNAMP case by Eq. (15). In 
the HFB case we solve Eq. (9) but without the PN projector and in HFB+PNAMP case by Eq. (15) using the corresponding intrinsic wave functions. The wave functions used to calculate the HFB surface are neither eigenfunctions of the particle number nor of the angular momentum. Those of the PNVAP conserve the particle number but not the angular momentum. Finally, the ones of the HFB+PNAMP and PNVAP+PNAMP conserve both symmetries. The angular momentum and parity $I=1 / 2^{+}$corresponds to the ground state of this nucleus (see Ref. ([17]) for a detailed description). The HFB and PNVAP approaches predict an axially symmetric ground state $(\beta \approx$ 0.3 ) though rather soft in $\gamma$. The angular momentum projection shifts the minimum to a triaxial shape and a larger deformation $(\beta \approx 0.4)$. The HFB+PNAMP minimum is more triaxial $\left(\gamma=30^{\circ}\right)$ than the PNVAP+PNAMP one $\left(\gamma=20^{\circ}\right)$ and softer in the oblate direction. To analyze the
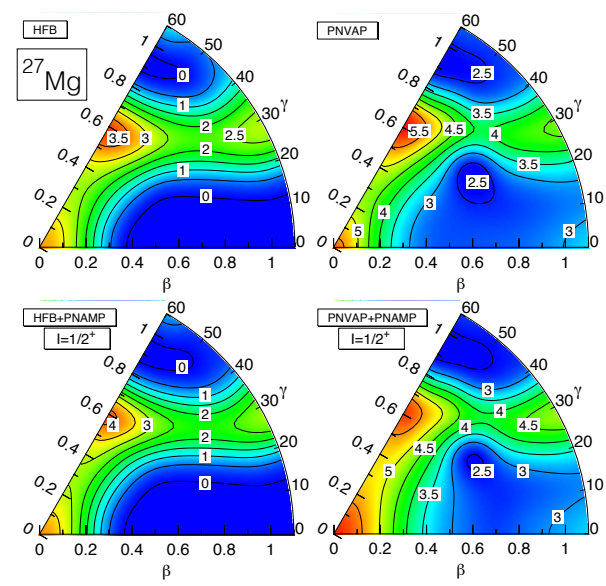

Figure 2. Contour plots of the absolute value of the proton pairing energies in the $(\beta$, gamma $)$ plane in several approaches. The color code is panel dependent.

quality of the HFB approach in the $(\beta, \gamma)$ plane we now study the pairing correlations in the different approaches, see Ref. [26] for a precise definition in BMF approaches. In Fig. 2 we show the absolute value of the pairing energies for protons, in the top panels in the HFB and the PNVAP approaches. The most striking aspect is that in the HFB case the pairing correlations vanish or are very small in large areas of the $(\beta, \gamma)$ plane at variance with the PNVAP approach where the smallest value is $2.5 \mathrm{MeV}$. At first glance it may be surprising that, though quantitatively very different, the pairing energies in the HFB and the PNVAP approach look qualitatively similar. This is however expected, since pairing correlations are primarily determined by the level density and this is fixed by the $(\beta, \gamma)$ deformation parameters. In the lower panels of Fig. 2 the projected versions are displayed. In the PNVAP+PNAMP by comparison with the PNVAP panel we can observe the effect of angular momentum projection. Though the PNVAP+PNAMP provides somewhat larger pairing than the PNVAP in some areas, both plots are very similar. This result is for $I=1 / 2^{+}$. For larger values of the angular momentum greater deviations are expected. In the HFB+PNAMP case where we can learn about the effect of the PNP after the variation by comparison with the HFB panel we find that there are only small changes. This is to be expected since in the HFB case the pairing correlations are either zero or rather small.
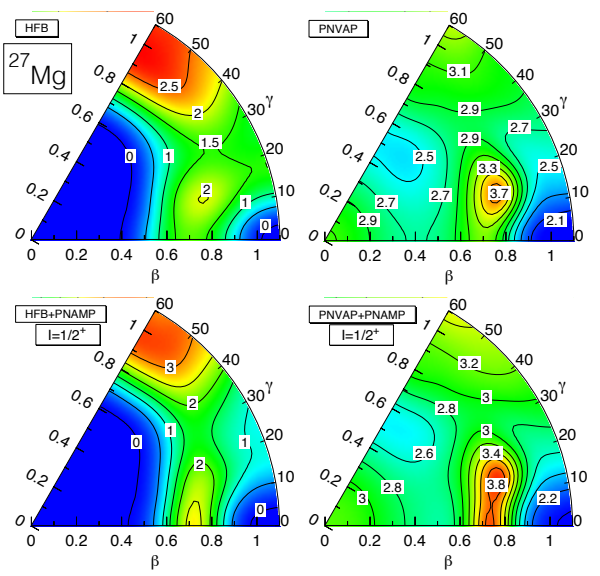

Figure 3. Same as Fig. 2 for neutrons.

In the neutron channel we anticipate a generalized lowering of the pairing correlations due to the blocked neutron. This can be observed in Fig. 3 where the neutron pairing energies are represented. We find that even in the PNVAP case the pairing correlations are reduced by about $30 \%$ as compared with the protons. In the HFB case we find again large areas where the pairing collapse takes place. We also observe that in this case the qualitative resemblance found in the proton channel between the HFB and the PNVAP pairing correlations is less pronounced. Apparently the VAP treatment is able to change the blocked structure as compared with the HFB one. We can appreciate the impact of the blocking effect by looking at the neutron energies in the even-even nucleus ${ }^{28} \mathrm{Mg}$ shown in Fig 4. Here we find smaller pairing energies in HFB as compared with the PNVAP approach, but there is a big difference as compared with ${ }^{27} \mathrm{Mg}$, namely that the areas where the pairing collapse takes place are much smaller.

It is important to notice, by looking at the HFB+PNAMP panel of Fig. (1), that in large areas and in particular around the energy minimum the pairing correlations for protons and neutrons are either zero or small, at variance with the PNVAP+PNAMP approach. This will have a great impact on the SCCM approaches, mentioned in the Introduction, used for full spectroscopy [9]. In these approaches the wave function is given by

$$
\left|\Psi_{M, \sigma}^{N, I, \pi}\right\rangle=\sum_{K, \beta, \gamma} g_{K \sigma}^{I}(\beta, \gamma) P^{N} P_{M K}^{I}\left|\tilde{\phi}^{\pi}(\beta, \gamma)\right\rangle .
$$




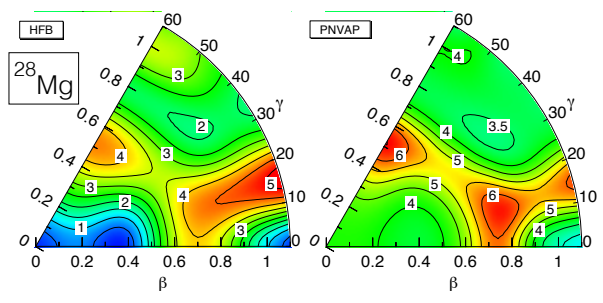

Figure 4. Neutron pairing energies for ${ }^{28} \mathrm{Mg}$ in the HFB and the PNVAP approches.

That means the previous states $\left|\tilde{\phi}^{\pi}(\beta, \gamma)\right\rangle$ are considered as basis states. The collapse of the pairing correlation for many $(\beta, \gamma)$ values will have a strong impact in SCCM calculations not only locally, as in the SCMF calculations, but also on the dynamical correlations (i.e. the associated masses). To gauge this impact we have performed some calculations in the simpler SCMF approach. Though we know that in the SCMF theory additional correlations are missing for a good description of excited states, to investigate the impact of a proper treatment of the pairing correlations on the spectrum we now solve Eq. (12) for spin values $1 / 2,3 / 2,5 / 2, \ldots$ and positive and negative parity in the HFB+PNAMP and PNVAP+PNAMP cases. The corresponding spectrum is plotted in Fig. 5. Though the level ordering is the same in both approximations we find large differences in the spacing. In particular we find that the HFB+PNAMP provides a much more compressed spectrum as the PNVAP+PNAMP approach.

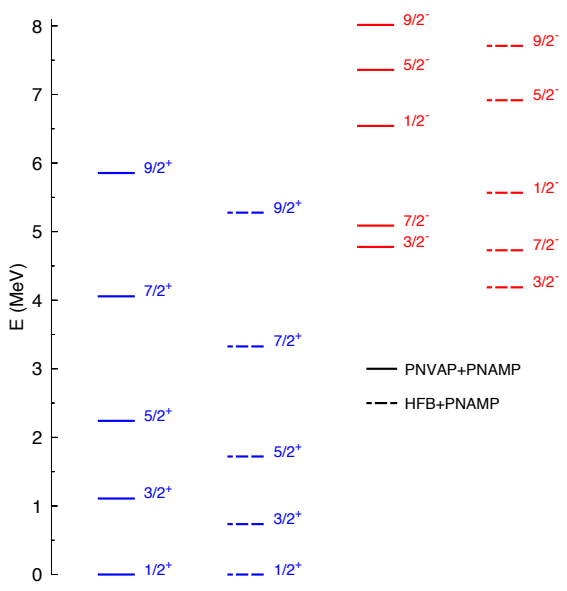

Figure 5. Spectrum of the nucleus ${ }^{27} \mathrm{Mg}$ in the HFB+PNAMP and the PNVAP+PNAMP.

In conclusion, we have presented a study of the pairing correlations in odd-A nuclei in the framework of the symmetry conserving mean field approximation with the effective Gogny force. We have analyzed for ${ }^{27} \mathrm{Mg}$ the pairing correlations in the $(\beta, \gamma)$ plane with the striking result that in the HFB approach they collapse or are very small for many points of the grid, in contrast to the variation after projection approach. We illustrate the difference of both approaches in the case of the excitation spectrum.

\section{Acknowledgements}

This work was supported by the Spanish Ministerio de Economía y Competitividad under contract FPA201457196-C5-2-P.

\section{References}

[1] Fifty Years of Nuclear BCS R. A. Broglia and V. Zelevinsky Eds. World Scientific, Singapore, 2013.

[2] H.J. Mang, Phys. Rep. 18C (1975) 325.

[3] J.L. Egido, P. Ring, Nucl. Phys. A388, 19-36 (1982)

[4] E. Hammarén, K. W. Schmid, F. Grümmer, A. Faessler, and B. Fladt, Nucl. Phys. A437, 1 (1985).

[5] P. Ring and P. Schuck, The Nuclear Many Body Problem (Springer-Verlag, Berlin, 1980).

[6] M. Bender, P.-H. Heenen and P.-G. Reinhard, Rev. Mod. Phys. 75 (2003) 121.

[7] T. Nikišć, D. Vretenar and P. Ring, Prog. Part. Nucl. Phys. 66 (2011) 519.

[8] J.L. Egido, Phys. Scr. 91 (2016) 073003.

[9] J. L. Egido, M.Borrajo, T. R. Rodríguez, Phys. Rev. Lett. 116, 052502 (2016)

[10] M. Bender and P.-H. Heenen, Phys. Rev. C 78 (2008) 024309.

[11] T.R. Rodríguez and J.L. Egido, Phys. Rev. $\mathbf{C 8 1}$ (2010) 064323.

[12] J. M. Yao, J. Meng, P. Ring, and D. Vretenar, Phys. Rev. C81 (2010) 044311.

[13] N. López Vaquero, T. R. Rodríguez and J. Luis Egido, Phys. Lett. B704 (2011) 520.

[14] N. López Vaquero, J.L. Egido and T. R. Rodríguez Phys. Rev. C 88 (2013) 064311.

[15] N. López Vaquero, T. R. Rodríguez and J.L. Egido Phys. Rev. Lett. 111 (2013) 142501.

[16] B. Bally, B. Avez, M. Bender, and P.-H. Heenen, Phys. Rev. Lett. 113, 162501 (2014).

[17] M. Borrajo and J.L. Egido, Eur. Phys. J. A52 (2016) 277.

[18] M. Borrajo and J.L. Egido, Physics Letters B 764 (2017) 328-334

[19] J. Dechargé and D. Gogny, Phys. Rev. C 21 (1980) 1568.

[20] J. F. Berger, M. Girod and D. Gogny, Comp. Phys. Commun. 63 (1991) 365.

[21] J.L. Egido, H.J. Mang, P. Ring, Nucl. Phys. A334 (1980) 1

[22] J.L. Egido, J. Lessing, V. Martin, L.M. Robledo, Nucl. Phys. A 594 (1995) 70.

[23] J.J. Griffin and J.A. Wheeler, Phys. Rev. 108, 311 (1957)

[24] M. Anguiano, J.L. Egido and L. M. Robledo, Nucl. Phys. A 683(2001) 227. 
[25] R. Rodriguez-Guzman, J. L. Egido, L. M. Robledo, Nucl. Phys. A 709, 201(2002)
[26] M. Anguiano, J.L. Egido, L.M. Robledo Phys. Lett. B 545 62-72 (2002) 